\title{
Development of time-resolved immunofluorometric assays for the detection of house dust mite-allergic IgE in human sera
}

\author{
Ratchanoo Phiphatchaipaisarn ${ }^{1}$, Jundee Rabablert ${ }^{1 *}$, Kornkarn Bramarapravati ${ }^{2}$, \\ Duangthep Thongdee $^{1}$, Nares Wongpitoon ${ }^{3}$, Worawan Durongpisitkul ${ }^{3}$, Nat Malainual ${ }^{4}$ \\ ${ }^{1}$ Department of Biology, Faculty of Science, Silpakorn University, Nakorn Pathom, Thailand; *Corresponding Author: jundee@su.ac.th; \\ ${ }^{2}$ Department of Preclinical Science, Faculty of Medicine, Thammasat University, Patumthani, Thailand; \\ ${ }^{3}$ Department of Pediatric, Police General Hospital, Bangkok, Thailand; \\ ${ }^{4}$ Department of Parasitology, Faculty of Medicine Siriraj Hospital, Mahidol University, Bangkok, Thailand.
}

Received 6 September 2010; revised 15 September 2010; accepted 16 September 2010.

\begin{abstract}
Dermatophagoides farinae and $D$. pteronyssinus are the prevalent house dust mites (HDM) in tropical countries and are associated with allergic diseases. This investigation developed a timeresolved immunofluorometric assay (TR-IFMA) for the first time to detect specific IgE antibody in patients with skin prick test positive to HDM but no detectable IgE by other means. Levels of IgE to natural and recombinant HDM allergens were measured by TR-IFMA in $50 \mathrm{HDM}$-allergic patients and 19 healthy participants compared to sandwich enzyme-linked immunosorbent assay (ELISA). A recombinant allergen, rDerf2, showed a $14 \mathrm{kDa}$ band corresponding to broad range proteins of natural HDM.TR-IFMA showed sensitivity lower than $0.35 \mathrm{kUA} / \mathrm{l}$. TR-IFMA employing three HDM antigens showed good correlations with sandwich ELISA at R2 0.93-0.96. TR-IFMA detected HDM IgE in 62, 62, 25 percent of allergic patient serum sample compared to 28 , 32 , and 22 percent detected by ELISA result using three HDM allergen. TR-IFMA also detected 26.3, 31.6, and 5.3 percent positive samples from 19 healthy participants while ELISA showed $0,5.3$, and 0 percents $\lg E$ positive samples. The use of rDerf2 as an HDM allergen for the assay was verified with no statistically different from other HDM allergens. TR-IFMA showed lower detection limit than ELISA and yielded higher sensitivity for serum of people with allergic symptoms with no detectable HDM IgE. It is anticipated that TR-IFMA for HDM-specific IgE detection will play an important role in future diagnosis of HDM allergy in clinical laboratories and for
\end{abstract}

different research purposes.

Keywords: House Dust Mites; Time-Resolved Immunofluorometric Assay; Allergy; IgE

\section{INTRODUCTION}

House-dust mites (HDMs) represent one of the most important allergen sources for the development of allergic diseases worldwide, particularly asthma and allergic rhinitis [1]. Dermatophagoides farinae (Df, originally known as American HDM) and Dermatophagoides pteronyssinus (Dp, European HDM) are the predominant species in tropical and subtropical climates [2]. More than $80 \%$ of allergic patients are sensitized to mite allergens $[3,4]$.

Positive skin prick tests (SPT) or serum IgE antibodies to HDMs are demonstrable in allergic patients. However, if patients are allergic to histamine, skin prick test could lead to complicated immunoreaction harmful to the patients [5]. Enzyme-linked immunosorbent assay (ELISA) is the method used to quantify serum HDM-IgE in vitro in laboratories [6]. However, a more sensitive in vitro test is needed for people who have dust mite allergic clinical diagnosis but shown undetectable HDM-IgE in their serum.

Monoclonal antibodies (mAbs) have been frequently used in allergy research. Their uses include the quantification of environmental allergen [7], allergens purification [8], and crystal structure [9]. Specificity for unique epitopes and unlimited in vitro production capability are the advantage of allergen-specific mAbs.

The purpose of this study was to development a timeresolved immunofluorometric assay (TR-IFMA) for the measurement of HDM-specific IgE. The TRF is characterized by lower detection limits and greater specificity, 
reproducibility and practicability [10]. Two group II HDM allergens and a group two recombinant vector were used for validation of the assay.

\section{METERIALS AND METHODS}

\subsection{Reagents}

Commercial allergens were purchased from Allertech (ALK, UK). DELFIA ${ }^{\circledR}$ Wash, DELFIA ${ }^{\circledR}$ Assay Buffer, DELFIA $^{\circledR}$ EU-labeled Streptavidin and DELFIA ${ }^{\circledR}$ Enhancement Solution were purchased from Perkin-Elmer Life Sciences (Wallac Oy, Turku, Finland). Monoclonal anti-group 2 antibodies (mAb 1D8 clone, Indoor biotecnologies Ltd, Manchester, UK). Biotinylated rabbit-anti mouse IgG solution and Biotinylated-labeled Mouse anti-human IgE antibody purchased from SouthernBiotech (Birmingham, USA). BCIP/NBT 1 Component Substrate and $\mathrm{ABTS}{ }^{\circledR}$ Peroxidase 1 Component substrate Solution were purchased from KPL (MD, USA). C96 MicroWell $^{\circledR}$ plates were purchased from Nunc ${ }^{\mathrm{TM}}$ (Roskilde, Denmark). The instrument used for measurement - DELFIA 1420 Automatic Immunoassay System - is the product of Perkin-Elmer Life Sciences (Wallac Oy, Turku, Finland).

\subsection{HDM Extracts and Recombinants Mite Allergens}

Total HDM extracts of $D$. farinae (Df) and D. pteronyssinus $(\mathrm{Dp})$ were prepared from Spent Mite medium from Siriraj Dust Mite Center, Bangkok, Thailand [7]. The extracts were stored at $-20^{\circ} \mathrm{C}$.

Recombinant rDerf2 allergen was constructed with ligated cDNA in pPICZ vector. It was a kind gift from Assist. Prof. Dr. Surapon Piboonpocanun, the Institute of Molecular Biology and Genetics, Mahidol University, Thailand. The positive transformants were subjected to expression in Pichia pasroris $[11,12]$. The supernatants were stored at $-20^{\circ} \mathrm{C}$ until used.

Proteins were separated by SDS-PAGE followed by transferring the fractionated proteins to a nitrocellulose membrane using method as described by the manufacturer with a wet blotting apparatus (Bio-Rad, USA). The blotted membrane was blocked overnight at $4{ }^{\circ} \mathrm{C}$. The membrane was further incubated with Monoclonal antigroup 2 antibodies, followed by Biotinylated rabbit-anti mouse IgG solution (1:5000) and BCIP/NBT 1 Component Substrate.

\subsection{Immunological Analysis}

\subsubsection{Enzyme-Linked Immuosorbent Assay (ELISA) \\ Specific IgE to Df, rDerf2 and Dp were analyzed by}

the ImmunoCAPTM system by using the cut-off limit 0.35 kilo units of aprotinin-specific antibodies per litre $\left(\mathrm{kU}_{\mathrm{A}} / \mathrm{l}\right)$, as recommended by the manufacturer. They were analyzed by sandwich ELISA (will be referred to as ELISA thereafter) as previously described [13]. Wells were coated with $1 \mathrm{D} 8$ clone $\mathrm{mAb}$ and let incubated over-night. Then $1 \mathrm{ng} /$ well allergens in PBS containing 1\% BSA were added. The wells were further incubated with serum $(1: 10)$, followed by biotinylated-labeled Mouse anti-human IgE antibody (1:1000), streptavidin-peroxidase and ABTS ${ }^{\circledR}$ Peroxidase 1 Component substrate. The result was measured in a DELFIA 1420 Automatic Immunoassay System.

\subsubsection{Time-Resolved Immunoflurometric Assay (TR-IFMA)}

Specific IgE to all three allergens were analyzed by the ImmunoCAPTM system by using the cut-off limit $0.35 \mathrm{kU}_{\mathrm{A}} / 1$ as recommended by the manufacturer. Df, recombinant Derf2 and Dp were analyzed by TR-IFMA [13]. Wells were coated with $\mathrm{mAb}$ and incubated overnight. After incubation, $1 \mathrm{ng} /$ well allergens in PBS containing $1 \%$ BSA were added. The wells were further incubated with serum $(1: 10)$, followed by biotinylatedlabeled Mouse anti-human IgE antibody (1:1000), DELFIA $^{\circledR}$ EU-labeled Streptavidin and DELFIA ${ }^{\circledR}$ enhancement solution. The result was measured in a DELFIA 1420 Automatic Immunoassay System.

\subsection{Patients and Collection of Samples}

Fifty rhinitis patients with/without intermittent or persistent, mild-to-moderate asthma with positive result to Skin Prick Test (SPT) were selected for this investigation by Dr. Nares Wongpitoon or Dr. Voravan Durongpisitkul of Department of Pediatric, Police General Hospital, Bangkok, Thailand. As the control group, 19 healthy subjects with no history of allergic diseases were included. The study was approved by the Ethics committee of the Police general Hospital and written informed consent was obtained from all participants.

Five milliliter of venous blood was taken from each participant. The blood was centrifuged within $6 \mathrm{hr}$ of collection at $400 \mathrm{~g}$ for $30 \mathrm{~min}$. The serum was then collected and stored frozen at $-20^{\circ} \mathrm{C}$.

In order to quantify specific IgE levels, serum from three highest HDM-sensitized individuals as analyzed by ELISA and TR-IFMA were analyzed by the Immuno$\mathrm{CAP}^{\mathrm{TM}}$ system at the laboratory of Faculty of Medicine, Mahidol University, Bangkok, Thailand. The IgE levels in several dilutions of the reference serum were analyzed. Fifth calibrator points in two-fold dilutions ranging $0.22-3.49,0.22-3.49$ and $0.28-4.55 \mathrm{kU}_{\mathrm{A}} / 1$ of $\mathrm{Df}, \mathrm{rDerf} 2$ and $\mathrm{Dp}$ specific IgE respectively were made for use in 
calibrating curve of both ELISA and TR-IFMA.

\subsection{Statistical Analysis}

Levels of specific IgE to HDM allergens in ELISA and TR-IFMA were obtained from Version 13 (Chicago, IL, USA).

\section{RESULTS}

\subsection{HDM-Allergen Characteristics}

HDM-allergen characteristics were determined by SDS-PAGE and Immunoblot using monoclonal antibody, as shown in Figure 1. Result from SDS-PAGE showed the MW of Df and Dp ranged from 10 to $72 \mathrm{kDa}$, while the molecular weight of rDerf2 was approximately $14 \mathrm{kDa}$. Immunoblot also confirmed rDerf2 molecular weight at $14 \mathrm{kDa}$.

\subsection{Calibration Curve and Detection Limit}

A typical standard curve for HDM-specific IgE using ELISA and TR-IFMA is shown in Figure 2. The sensitivity ( \pm 2 standard deviations) of the assay, as calculated from $\mathrm{X}$ replicates of the zero standards, was about $<$ $0.35 \mathrm{kU}_{\mathrm{A}} / 1$. The calibration curve was linear.

\subsection{Subject Characteristics}

Specific antibodies to HDM allergens were examined in a total of 69 subjects. Subjects were screened by SPT using commercial Der f, Der p, CR, Grass, Dog, Cat, Wheat, Shrimp, Feather, M1 and M2. The demographic clinical characteristics of the subjects were shown in Table 1.

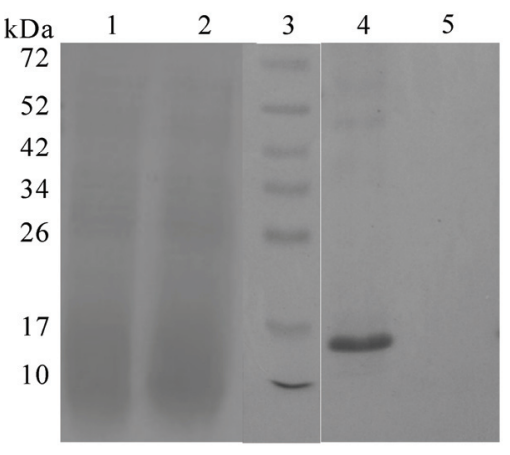

(a)

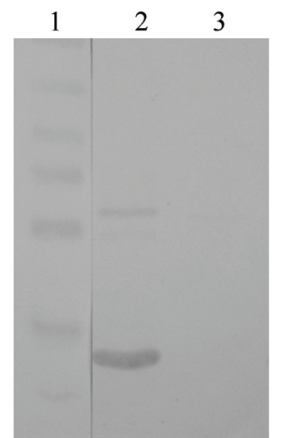

(b)
Figure 1. The protein components of HDM allergen were determined by SDS-PAGE (a); Lane 1: Df; Lane 2: Dp; Lane 3: Molecular mass standards (in $\mathrm{kDa}$ ); Lane 4: rDerf2; Lane 5: pPICZ. (b) Immunoblot Lane 1: Molecular mass standards (in $\mathrm{kDa}$; Lane 2: rDer f 02; Lane 3: pPICZ.

\subsection{Comparison of TR-IFMA and ELISA}

Number of positive samples from ELISA and TRIFMA in allergic patient and healthy participant serum as seen using Df, rDerf2 and Dp allergen against group II $\mathrm{mAb}$ is shown in Table 2. TR-IFMA detected HDM IgE in higher percentage of allergic patient serum sample when tested using Df, rDerf2 and Dp allergen $(62,62,25$ percent) compared to ELISA result $(28,32$, and 22 percent respectively). In 19 healthy subjects, ELISA detected HDM IgE in 0, 5.3, and 0 percents samples tested by the three HDM allergens while TR-IFMA found 26.3, 31.6, and 5.3 percent positive samples respectively.

TR-IFMA was compared to ELISA by parallel testing of 50 serum specimens from rhinitis patients utilizing Df, rDerf2 and Dp allergen respectively. Among allergic patients very good overall correlation was found (Figure 3). The linear regression coefficient values calculated from the positive specimens varied from 0.93-0.96.

Table 1. Demographic and characteristics of the study individuals.

\begin{tabular}{ccc}
\hline \multirow{2}{*}{ Characteristics } & \multicolumn{2}{c}{ Groups } \\
\cline { 2 - 3 } & $\begin{array}{c}\text { allergic } \\
\text { individuals (A) }\end{array}$ & $\begin{array}{c}\text { non-allergic } \\
\text { individuals (B) }\end{array}$ \\
\hline Number of subjects (n) & 50 & 19 \\
Age (years, mean \pm SD) & $39.52 \pm 9.93$ & $25.21 \pm 6.9$ \\
Gender (male/female) & $24 / 26$ & $4 / 15$ \\
Clinical diagnosis (N, \%) & $50,100 \%$ & N/A \\
Catagorized by allergen (\%) & & \\
Der f & $100 \%$ & \\
Der p & $100 \%$ & \\
CR & $20 \%$ & \\
Grass & $8 \%$ & \\
Dog & $4 \%$ & \\
Cat & $8 \%$ & \\
Wheat & $0 \%$ & \\
Shrimp & $12 \%$ & \\
Feather & $0 \%$ & \\
M1 & & \\
M2 & N & \\
\hline
\end{tabular}

Der p, Dermatophagoides pteronyssinus; Der f, Dermatophagoides farinae; $\mathrm{CR}$, American Cockroach; Grass, Bermuda grass pollen; Dog, Dog epithelium; Cat, Cat pelt; Feather, feather of Chicken, Duck, and Goose; M1, Penicillium notatum, Aspergillus niger, Drechslera, Cladosprium spharospermum, Alternaria; M2, Curvularia, Fusarium, Aureobasidium, Mucor, Rhizopus 


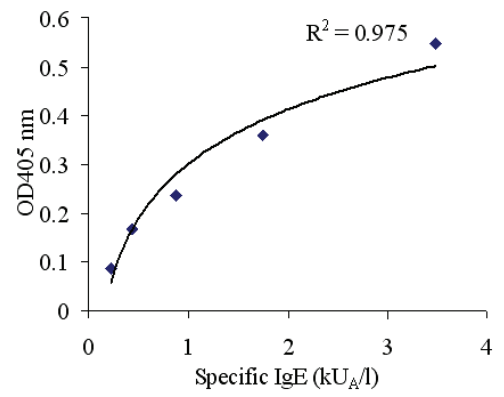

(a)

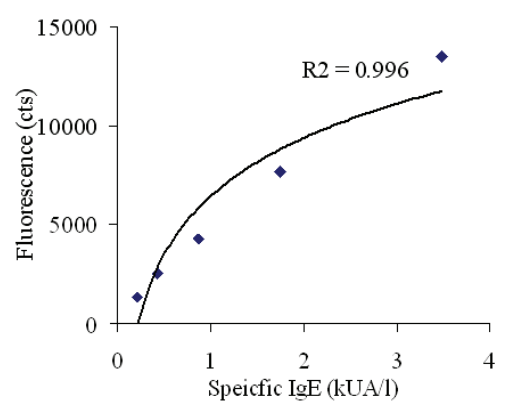

(d)

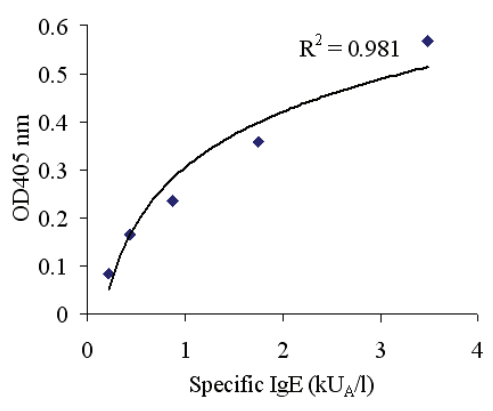

(b)

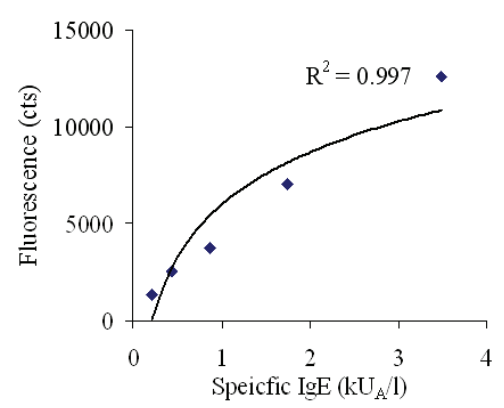

(e)

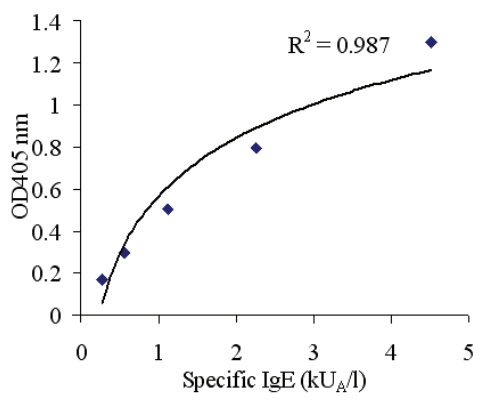

(c)

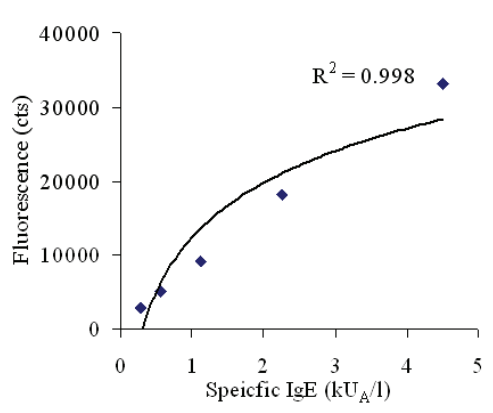

(f)

Figure 2. Standard curve for sandwiched ELISA for (A) Df, (B) rDerf2, (C) Dp. Standard curve for TR-IFMA for (D) Df, (E) rDerf2, (F) Dp. Each point on the standard curve is the mean of 3 duplicate measurements.

Table 2. Number of positive samples from ELISA and TRIFMA in allergic patient and healthy participant serum as seen using Df, rDerf2 and Dp allergen against group II mAb.

\begin{tabular}{|c|c|c|}
\hline \multirow[b]{2}{*}{ Allergen tested } & \multicolumn{2}{|c|}{ Number of positive samples: $\mathrm{n}$ (frequency \%) } \\
\hline & $\begin{array}{l}\text { Allergic patients (A) } \\
\text { Total } n=50\end{array}$ & $\begin{array}{c}\text { Healthy participants (B) } \\
\text { Total } n=19\end{array}$ \\
\hline \multicolumn{3}{|l|}{$\mathrm{Df}$} \\
\hline ELISA & $14(28)$ & $0(0)$ \\
\hline TR-IFMA & $31^{1}(62)$ & $5(26.3)$ \\
\hline \multicolumn{3}{|l|}{ rDerf2 } \\
\hline ELISA & $16(32)$ & $1(5.3)$ \\
\hline TR-IFMA & $31^{2}(62)$ & $6(31.6)$ \\
\hline \multicolumn{3}{|l|}{$\mathrm{Dp}$} \\
\hline ELISA & $11(22)$ & $0(0)$ \\
\hline TR-IFMA & $25^{3}(50)$ & $1(5.3)$ \\
\hline
\end{tabular}

${ }^{1,2}$ and ${ }^{3}$ correspond to the same data in Table 3.

TR-IFMA positive cases from serum of allergic patients tested with all three HDM allergen were separated into two groups, namely those from ELISA positive and ELISA negative samples. Number of samples, frequency and median of each group is shown according to specific allergen in Table 3. Median, standard deviation, 25 and 75 percent values are presented in Figure 4. Among TRIFMA positive samples, 54.84, 48.39, and 56 percents of Df, rDerf2, and Dp were identified as ELISA negative samples.

Concentrations of HDM IgE TR-IFMA from all three HDM allergens testing (allergic patients and healthy participants) were subjected to test for several related sample using Friedman test. At $\mathrm{N}=69, p$ value equals 0.093 . The use of any of the three HDM allergens does not give a statistically significant result in TR-IFMA technique.

\section{DISCUSSIONS}

It has been reported that D. farinae which homologous with $D$. pteronyssinus is composed of broad range of 10 to $72 \mathrm{kDa}$. In the present study, Immunoblotting analysis of rDer f 2 using a specific $\mathrm{mAb}$ showed one band of $14 \mathrm{kDa}$ (Figure 1), agreeing with previous reports and indicating that rDer f 2 corresponded to $D$. farinae $[14,15]$. It is known that $D$. farinae usually coexits with $D$. pteronyssinus. As a result the assay measuring IgE binding to Derf2 would not distinguish $D$. farinae sensitization with cross reactivity [16]. 


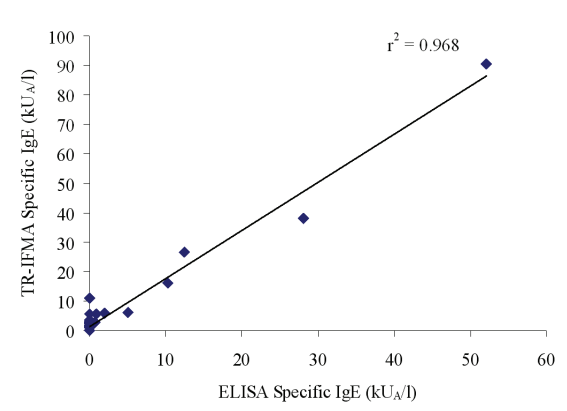

(a)

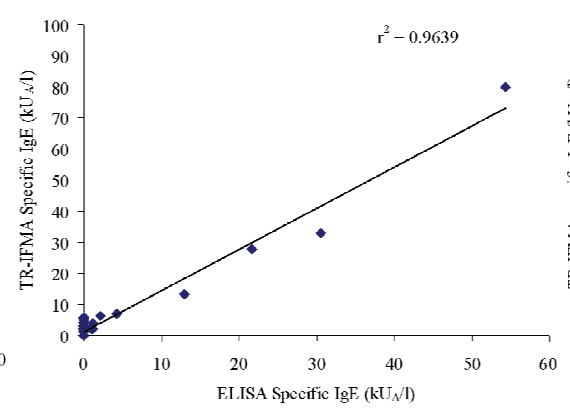

(b)

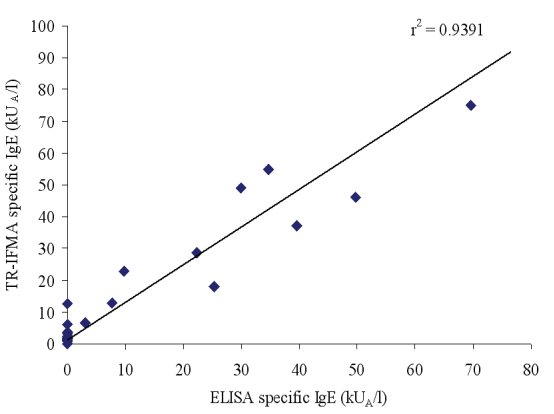

(c)

Figure 3. Correlation between levels of specific IgE against Df (a), rDerf2 (b) and Dp (c) measured by ELISA and TR-IFMA.

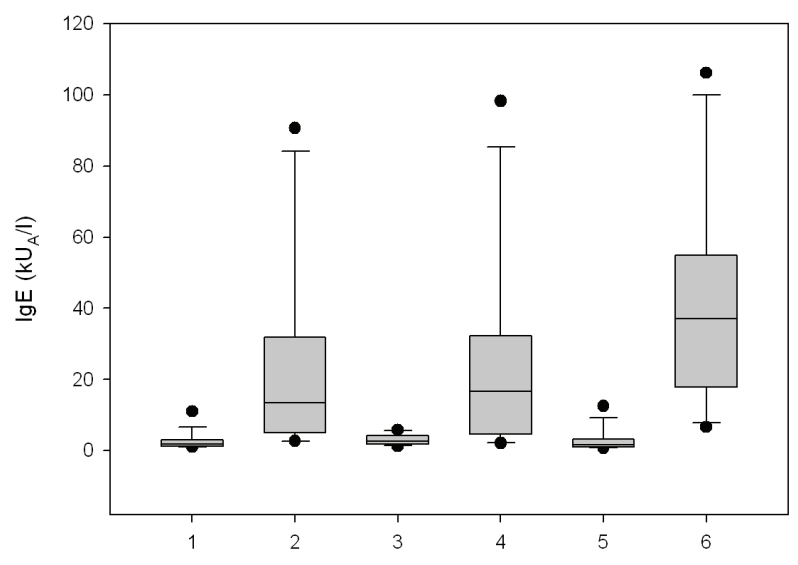

Figure 4. TR-IFMA positive cases from serum of allergic patients tested with all three HDM allergens. Median, standard deviation, 25 and 75 percent values are presented. HDM allergens Df: 1) ELISA negative 2) ELISA positive rDerf2: 3) ELISA negative 4) ELISA positive Dp: 5) ELISA negative 6) ELISA positive.

\subsection{Levels of Specific IgE Reactivity to HDM Allergens: Lower Detection Limit and Increase Testing Sensitivity}

The IgE reactivity profile for the sera from allergic patients $(\mathrm{n}=50)$ and healthy participants $(\mathrm{n}=19)$ was determined by three different allergens, namely Df, rDer f 2 and Dp measured by TR-IFMA compared with ELISA.

As shown in Table 2, the efficacy of recombinant antigen (rDerf 2) and natural antigen (Df or Dp) were examined in allergic patients and non-allergic individuals by both TR-IFMA and ELISA. In allergic patients, we found that only 28,32 , and 22 percents result was obtained by ELISA, while 62, 62, and 50 percent of patient serum reacted with Df, rDerf2, and Dp respectively using TR-IFMA. The increase in rate of HDM IgE detection was significant at 34,30 and 28 percents for all three allergen respectively. This is the first indication
Table 3. TR-IFMA positive cases in serum from allergic patients categorized by ELISA result in Df, rDerf2 and Dp testing.

\begin{tabular}{|c|c|c|}
\hline \multirow[b]{2}{*}{ HDM allergens } & \multicolumn{2}{|c|}{$\begin{array}{l}\text { TR-IFMA positive cases in } \\
\text { symptomatic patients }\end{array}$} \\
\hline & ELISA positive & ELISA negative \\
\hline \multicolumn{3}{|l|}{ Df: total 31 samples ${ }^{1}$} \\
\hline n (frequency \%) & $14(45.16)$ & $17(54.84)$ \\
\hline Median $\left(\mathrm{kU}_{\mathrm{A}} / \mathrm{l}\right)$ & 13.37 & $1.81^{*}$ \\
\hline \multicolumn{3}{|l|}{ rDerf2: total 31 samples $^{2}$} \\
\hline n (frequency \%) & $16(51.61)$ & $15(48.39)$ \\
\hline Median $\left(\mathrm{kU}_{\mathrm{A}} / \mathrm{l}\right)$ & 16.67 & $2.74 *$ \\
\hline \multicolumn{3}{|l|}{ Dp: total 25 samples $^{3}$} \\
\hline n (frequency \%) & $11(44)$ & $14(56)$ \\
\hline $\operatorname{Median}\left(\mathrm{kU}_{\mathrm{A}} / \mathrm{l}\right)$ & 37.19 & $1.66^{*}$ \\
\hline
\end{tabular}

Mann-Whitney Rank Sum Test; $p<0.001 *$

suggesting that TR-IFMA of all three HDM allergens show higher sensitivity for serum of people with allergic symptoms with no detectable HDM IgE. In addition, when ELISA showed 0, 5.3, and 0 percent positive samples in healthy participant serum, TR-IFMA detected $26.3,31.6$, and 5.3 percent positive samples. That is the 26.3, 21.3 and 5.3 percent higher detection rate across the board.

In $50 \mathrm{HDM}$-SPT positive allergic subjects, 48 percents of this group showed cross-reactive IgE binding to all three allergens measured by TR-IFMA, while only 22 percent showed cross-reactive IgE binding to all three allergens measured by ELISA. Additionally, cross-reactive IgE to two allergens was also observed by both TRIFMA and ELISA. TR-IFMA showed 10, 2 and 0 percent cross-reactivity between Df/rDerf2, rDerf2/Dp and Df/Dp, while ELISA result showed 2, 0 , and 0 percent respectively. In healthy participants there was no crossreactivity report over all three antigens. Only 15.8 and 5.3 
percent cross-reactivity were reported for Df/rDerf2 and rDerf2/Dp. These numbers confirms TR-IFMA sensitivity over ELISA, and suggests that rDerf2 is a slightly better antigen as seen from cross-reactivity data.

Table 3 and Figure 4 revealed lower detection limits and the sensitivity of TR-IFMA. All HDM allergens provided significantly different median between ELISA positive and negative samples in Mann-Whitney Rank Sum Test with $p<0.001$. Figure 4 shows that even though ranges of all three ELISA negative groups showed overlap with the ELISA positive ones, one hundred percent of all three ELISA negative populations stayed within 40 percentile of the corresponding ELISA positive population. TR-IFMA was able to showed real value of some ELISA negative samples beautifully in this demonstration.

This investigation employed three different HDM allergens, namely Df and Dp from natural sources, and a recombinant rDerf2. Result from the test for several related sample using Friedman test showed that TR-IFMA result using all three allergens was not significantly different from one another. Thus this investigation suggests that rDerf2 can be used as a HDM allergen in TR-IFMA. Since rDerf2 can be expressed in large quantity, this should ensure a homogeneous batch supply of HDM allergen for time to come.

Immunoassays based on time-resolved fluorometry represents an attractive option that offers several advantages over other traditional techniques, including very high sensitivity, no use of radioactive reagents, stability of the reagents, low background interference and a wide test range $[17,18]$. Additionally, the use of a specific $\mathrm{mAb}$ as capture antibody in this study avoided over estimation of allergens by reducing the cross-reactive epitopes recognition. The use of specific polyclonal serum avoided the loss of detection produced by conformational changes affecting one or more epitopes. Hence, a specific antiHDM polyclonal serum has been used as the secondary antibody in this investigation, unlike most of other immunoassays described [19].

The time-resolved immunofluormetric assay developed in this investigation provides sensitive and highly specific techniques for the determination of HDM-specific IgE in human sera. Measurements achieved with TRIFMA and ELISA were highly correlated (R2 between 0.93-0.96). In regard to the limit of detection, the assay allows the detection of HDM-specific IgE as low as < $0.35 \mathrm{kU}_{\mathrm{A}} / 1$, which is well below limits of detection reported by other techniques (Immunoblot, ELISA, RIA); TR-IFMA can therefore be considered as the most sensitive method for quantifying HDM-specific IgE. In addition, the assay ranges of TR-IFMA is the wider than those of ELISA in our laboratory. Our data was consis- tent with previous reports [20,21]. It is clearly seen that TR-IFMA showed sensitivity to serum of allergic patients with symptom whose serum were formerly HDM IgE negative using ELISA. The analysis of real samples from HDM positive allergic patients compared to HDM negative but positive to aeroallergen demonstrated that a broad range of HDM-specific IgE could be detected with the present techniques.

\section{CONCLUSIONS}

TR-IFMA was developed for HDM allergen IgE testing and evaluated. Such improvements may be useful for screening of HDM allergy and other aeroallergens. TRIFMA of all Df, rDerf2 and Dp showed lower detection limit than ELISA and yielded higher sensitivity for serum of people with allergic symptoms with no detectable HDM IgE. Recombinant HDM allergen, rDerf2, was proven to be as good an allergen as its natural counterpart, namely Df and Dp. It is anticipated that TR-IFMA for HDM-specific IgE detection will play an important role in future diagnosis of HDM allergy in clinical laboratories and for different research purposes.

\section{ACKNOWLEDGEMENTS}

This study was supported by the TRF (MRG-WII 505S061), a partial funding from Thailand Research Fund No. MRG-WII525S092 and DIG5180004. The authors thank Associate Professor Dr. Darawan Wanachiwanawin, Department of Parasitology, Faculty of Medicine Siriraj Hospital, Mahidol University for laboratory facility, and Assistant Professor Dr. Junya Pattaraarchachai, Faculty of Medicine, Thammasat University for statistic analysis.

\section{REFERENCES}

[1] Arruda, L.K., Rizzo, M.C., Chapman, M.D., FernandezCaldas, E., Baggio, D., Platts-Mills, T.A. and Naspitz, C.K. (1991) Exposure and sensitization to dust mite allergens among asthmatic children in São Paulo, Brazil. Clinical \& Experimental Allergy, 21(4), 433-439.

[2] Colloff, M.J. (1998) Distribution and abundance of dust mites within homes. Allergy, 53, 24-27.

[3] Nadchatram, M. (2005) House dust mites, our intimate associates. Tropical Biomedicine, 22(1), 23-37.

[4] Thomas, W.R., Smith, W.A. and Hales, B.J. (2004) The allergenic specificities of the house dust mite. Chang Gung Medical Journal, 27(8), 563-569.

[5] Williams, P., Sewell, W.A., Bunn, C., Pumphrey, R., Read, G. and Jolles, S. (2008) Clinical immunology review series: an approach to the use of the immunology laboratory in the diagnosis of clinical allergy. Clinical \& Experimental Immunology, 153(1), 10-18.

[6] Bush, R.K., Wood, R.A. and Eggleston, P.A. (1998) Laboratory animal allergy. Journal of Allergy and Clinical Immunology, 102(1), 99-112. 
[7] Chapman, M.D., Heymann, P.W., Wilkins, S.R., Brown, M.J. and Platts-Mills, T.A. (1987) Monoclonal immunoassays for major dust mite (Dermatophagoides) allergens, Der $\mathrm{p} I$ and Der f I, and quantitative analysis of the allergen content of mite and house dust extracts. Journal of Allergy and Clinical Immunology, 80(2), 184-194.

[8] Ferrandiz, R., Casas, R. and Dreborg, S. (1997) Purification and IgE binding capacity of Der s 3, a major allergen from Dermatophagoides siboney. Clinical \& Experimental Allergy, 27(6), 700-704.

[9] Suzuki, M., Tanaka, Y., Korematsu, S., Mikami, B. and Minato, N. (2006) Crystal structure and some properties of a major house dust mite allergen, Derf 2. Biochemical and Biophysical Research Communications, 339(2), 679686.

[10] Sheng, S.L., Bao, S.H., Huang, G. and Wang, L.M. (2008) Development of time-resolved immunofluorometric assays for vascular endothelial growth factor and application on plasma of patients with gastric tumours. Clinical \& Experimental Immunology, 151(3), 459-466.

[11] Piboonpocanun, S., Malainual, N., Jirapongsananuruk, O., Vichyanond, P. and Thomas, W.R. (2006) Genetic polymorphisms of major house dust mite allergens. Clinical \& Experimental Allergy, 36(4), 510-516.

[12] Tanyaratsrisakul, S., Malainual, N., Jirapongsananuruk, O., Smith, W.A., Thomas, W.R. and Piboonpocanun, S. (2009) Structural and IgE binding analyses of recombinant der p 2 expressed from the hosts escherichia coli and pichia pastoris. International Archives of Allergy and Immunology, 29(3), 190-198.

[13] Hales, B.J., Hazell, L.A., Smith, W. and Thomas, W.R. (2002) Genetic variation of Der p 2 allergens: effects on $\mathrm{T}$ cell responses and immunoglobulin $\mathrm{E}$ binding. Clinical \& Experimental Allergy, 32(10), 1461-1467.

[14] Koyanagi, S., Maeda, T., Murakami, T., Kawatsu, K., Sugawara, K., Miyatsu, Y. and Mizokami, H. (2008) Large-scale production of major house dust mite allergen der f 2 mutant (C8/119S) in escherichia coli. Journal of Bioscience and Bioengineering, 106(4), 387-392.

[15] Pittner, G., Vrtala, S., Thomas, W.R., Weghofer, M., Kundi M., Horak, F., Kraft, D. and Valenta, R. (2004) Component-resolved diagnosis of house-dust mite allergy with purified natural and recombinant mite allergens. Clinical \& Experimental Allergy, 34(4), 597-603.

[16] Yasueda, H., Mita, H., Yui, Y. and Shida, T. (1989) Comparative analysis of physicochemical and immunochemical properties of the two major allergens from Dermatophagoides pteronyssinus and the corresponding allergens from Dermatophagoides farinae. International Archives of Allergy and Applied Immunology, 88(4), 402407.

[17] Parra, M.D., Tuomola, M., Cabezas-Herrera, J. and Ceron, J.J. (2006) Analytical and clinical validation of a timeresolved immunofluorometric assay (TR-IFMA) for canine C-reactive protein in serum. Veterinary Research Communications, 30(2), 113-126.

[18] Yuan, A.S. and Gilbert, J.D. (1996) Time-resolved fluoroimmunoassay for the determination of lisinopril and enalaprilat in human serum. Journal of Pharmaceutical and Biomedical Analysis, 14(7), 773-781.

[19] Ferrandiz, R., Casas, R., Dreborg, S., Einarsson, R. and Fernandez, B. (1995) Crossreactivity between Dermatophagoides siboney and other house dust mite allergens in sensitized asthmatic patients. Clinical \& Experimental Allergy, 25(10), 929-934.

[20] Wu, F.B., Ouyan, H.Q., Tang, X.Y. and Zhou, Z.X. (2008) "Double-antigen sandwich time-resolved immunofluorometric assay for the detection of anti-hepatitis $\mathrm{C}$ virus total antibodies with improved specificity and sensitivity," Journal of Medical Microbiology, 57, 947-953.

[21] Sheng, S.L., Wang, Q. and Huang, G. (2007) Development of time-resolved immunofluorometric assays for CA 72-4 and application in sera of patients with gastric tumors. Clinica Chimica Acta, 380(1-2), 106-111. 